\title{
Effects of whole-body neuromuscular electrical stimulation device on hemodynamics, arrhythmia, and sublingual microcirculation
}

\author{
Megumi Hoshiai ${ }^{1} \cdot$ Kaori Ochiai $^{2} \cdot$ Yuma Tamura $^{2} \cdot$ Tomoki Tsurumi $^{2} \cdot$ Masato Terashima $^{2} \cdot$ Hajime Tamiya $^{2} \cdot$ \\ Eikou Maeno ${ }^{1}$ - Satoshi Mizuguchi ${ }^{1}$. Takashi Tomoe ${ }^{1}$. Atsuhiko Kawabe ${ }^{1}$ - Atsuko Uema ${ }^{1}$. Asuka Ueno ${ }^{1}$. \\ Takushi Sugiyama $^{1} \cdot$ Yasuto Horie $^{3} \cdot$ Hiroyuki Sugimura $^{3} \cdot$ Ryousuke Koike $^{4} \cdot$ Takanori Yasu $^{1}$
}

Received: 12 October 2020 / Accepted: 4 December 2020 / Published online: 6 February 2021

(c) The Author(s) 2021

\begin{abstract}
Neuromuscular electrical stimulation has been used to treat cardiovascular diseases and other types of muscular dysfunction. A novel whole-body neuromuscular electrical stimulation (WB-NMES) wearable device may be beneficial when combined with voluntary exercises. This study aimed to investigate the safety and effects of the WB-NMES on hemodynamics, arrhythmia, and sublingual microcirculation. The study included 19 healthy Japanese volunteers, aged 22-33 years, who were not using any medication. Electrocardiogram (ECG), echocardiography, and blood sampling were conducted before a 20-min WB-NMES session and at 0 and $10 \mathrm{~min}$ after termination of WB-NMES. Their tolerable maximum intensity was recorded using numeric rating scale. Arrhythmia was not detected during neuromuscular electrical stimulation or during $10 \mathrm{~min}$ of recovery. Blood pressure, heart rate, left ventricular ejection fraction, and diastolic function remained unchanged; however, mild mitral regurgitation was transiently observed during WB-NMES in a single male participant. A decrease in blood glucose and an increase in blood lactate levels were observed, but no changes in blood fluidity, sublingual microcirculation, blood levels of noradrenaline, or oxidative stress were shown. WB-NMES is safe and effective for decreasing blood glucose and increasing blood lactate levels without changing the blood fluidity or microcirculation in healthy people.
\end{abstract}

Keywords Arrhythmia $\cdot$ Oxidative stress $\cdot$ Rheology $\cdot$ Whole-body neuromuscular electrical stimulation $\cdot$ Microcirculation

\section{Introduction}

Exercise therapy is an inexpensive, safe, and extremely effective primary and secondary intervention for the prevention of cardiovascular and chronic respiratory diseases.

Megumi Hoshiai and Kaori Ochiai are joint first authors with equal contributions to this work.

Takanori Yasu

tyasu@dokkyomed.ac.jp

1 Department of Cardiovascular Medicine and Nephrology, Dokkyo Medical University Nikko Medical Center, Nikko, Tochigi, Japan

2 Department of Rehabilitation, Dokkyo Medical University Nikko Medical Center, Nikko, Tochigi, Japan

3 Department of Cardiology, Dokkyo Medical University Nikko Medical Center, Nikko, Tochigi, Japan

4 Department of Pulmonology, Dokkyo Medical University Nikko Medical Center, Nikko, Tochigi, Japan
Reduced exercise capacity after primary percutaneous coronary intervention in acute myocardial infarction patients may lead to worse clinical outcomes [1]. A major issue for rehabilitation in people with restricted movement is how to suppress skeletal muscle atrophy and the decreases in motor function $[2,3]$. It was suggested that the start date of gait is related to one of the predictors of activities of daily living in elderly people with heart failure with preserved ejection fraction [4]. Electrical muscle stimulation to encourage skeletal muscle contraction has been a known treatment for disuse-induced muscle atrophy. Even in patients diagnosed with New York Heart Association (NYHA) class IV heart failure, for whom exercise therapy is contraindicated, electrical stimulation can be safely used without affecting blood pressure or heart rate while increasing maximum muscle strength [5]. However, it is known to have important precautions and contraindications. For example, endocrine organs such as the thyroid and genitalia, sensory organs such as the eyes, and people with pacemakers are contraindicated for electrical muscle stimulation [5]. 
Several studies have used belt-electrode-type skeletal muscle electrical stimulation with electrodes attached to the torso, thighs, and lower legs to examine its effectiveness on circulatory dynamics, muscle contraction and hypertrophy, and safety in patients with arterial disease and diabetes mellitus [6-9]. Nowadays, there are novel and useful wearable devices for whole-body neuromuscular electrical stimulation (WB-NMES), rather than the traditional belt type, that efficiently provide electrical stimulation to the forearms and back arms, chest, back, abdomen, oblique abdomen, gluteus muscles, and front and back thigh muscles [10]. Watanabe et al. [10] have shown that a combination of aerobic exercise and WB-NMES for 15 min enhances metabolic response to the same extent as high-intensity exercise. It would be useful if this exercise regimen could be applied to patients with heart failure who cannot do vigorous exercise. However, the effect of battery-powered WB-NMES devices on hemodynamics, including of the heart and peripheral microcirculation, have not been fully elucidated. Blood fluidity plays an important role in the pathogenesis of various disease and is also a powerful predictor of cardiovascular events [11]. The factors behind the decrease in blood fluidity due to exercise are the transient increase in hematocrit, and the activation of leukocytes and platelets due to catecholamines and cytokines [12]. Strenuous exercise can lead to platelet activation, and hypercoagulation, which can lead to acute coronary syndrome [13]. We have reported that vigorous exercise induced transient deterioration in microcirculation [14]. There is an urgent need to evaluate the safety and effects of electrical stimulation to the whole body, including the chest, on circulatory dynamics, arrythmia, microcirculation, systemic metabolism including blood glucose, lactate levels, and oxidative stress.

This study aimed to examine the safety of a novel WBNMES wearable device as a modified phase 1 study and its effect on circulatory dynamics, arrythmia, sublingual microcirculation, blood glucose and lactate levels, and oxidative stress. This will help us evaluate whether it is safe to apply electricity using WB-NMES to the whole body including the chest.

\section{Materials and methods}

\section{Study participants}

The study participants comprised 19 healthy volunteers; non-smoking and non-obese Japanese men $(n=10)$ and women $(n=9)$ aged $22-33$ years. All were normotensive with body mass indices of $21-24 \mathrm{~kg} / \mathrm{m}^{2}$. No abnormalities were found during routine physical examinations and standard laboratory analyses after overnight fasting. Persons taking any medications were excluded. The study protocol was approved by the Ethical Committee of Dokkyo Medical University's Nikko Medical Center (Approval Number: Nikko 29012). All participants provided written informed consent for participation in this study. All procedures performed in this study complied with the national ethical guidelines for medical and health research involving human participants and with the 1964 Helsinki Declaration and its later amendments or comparable ethical standards. Baseline characteristics of the study participants regarding the routine physical examination and standard laboratory test results are shown in Table 1.

\section{Whole blood rheology}

We used a microchannel flow analyzer system (BWAMCFAN, Kikuchi Microtechnology Co., Ltd., Ibaraki, Japan) equipped with a microchannel array chip. Blood was drawn through BK 7-7-4.5 microchannels (Kikuchi Microtechnology Co., Ltd.) as an ex vivo model of microvessels to assess the whole blood rheology and leukocyte activity as previously described [15-17].Within $10 \mathrm{~min}$ of collecting blood into heparinized tubes, $0.1 \mathrm{~mL}$ of blood was drawn through BK 7-7-4.5 microchannels as an ex vivo capillary model (7854-parallel, $7 \times 4.5-\mu \mathrm{m}$ equivalent cross-section, $30 \mu \mathrm{m}$ long) under a constant vacuum of $20 \mathrm{~cm} \mathrm{H}_{2} \mathrm{O}$ $(1.96 \mathrm{kPa})$. The time required for saline to pass through the microchannels was determined before each blood measurement for calibration. The microscopic motion images of blood passing through the microchannels were monitored and stored on a computer system.

Table 1 Baseline haracteristics of the study participants

\begin{tabular}{ll}
\hline Characteristics & Total $(n=19)$ \\
\hline Age $(\mathrm{y})$ & $29 \pm 4$ \\
Male $(\%)$ & $10(52 \%)$ \\
Height $(\mathrm{cm})$ & $164.6 \pm 7.2$ \\
Weight $(\mathrm{kg})$ & $61.6 \pm 10.4$ \\
BMI $\left(\mathrm{kg} / \mathrm{m}^{2}\right)$ & $22.7 \pm 3.5$ \\
White blood cells $(/ \mu \mathrm{L})$ & $6257.1 \pm 1665.3$ \\
Hematocrit $(\%)$ & $44.0 \pm 3.1$ \\
Platelets $\left(\times 10^{3} / \mu \mathrm{L}\right)$ & $25.9 \pm 4$ \\
HDL-C $(\mathrm{mg} / \mathrm{dL})$ & $57.3 \pm 15$ \\
LDL-C $(\mathrm{mg} / \mathrm{dL})$ & $109.4 \pm 31.1$ \\
Triglycerides $(\mathrm{mg} / \mathrm{dL})$ & $97.8 \pm 45.4$ \\
Serum creatinine $(\mathrm{mg} / \mathrm{dL})$ & $0.7 \pm 0.2$ \\
Plasma glucose $(\mathrm{mg} / \mathrm{dL})$ & $84.4 \pm 5.8$ \\
HbA1c level $(\%)$ & $5.4 \pm 0.2$ \\
\hline
\end{tabular}

Data are expressed as the mean $\pm \mathrm{SD}$ (standard deviation)

$B M I$ body mass index, $H D L-C$ high-density lipoprotein cholesterol, $L D L-C$ low-density lipoprotein cholesterol, $H b A l c$ hemoglobin A1c 


\section{Sublingual microcirculation}

Sublingual microcirculation images were observed using a handheld dark-field CytoCam video microscope (Braedius Medical, Huizen, The Netherlands) [18] before and after the WB-NMES. The total blood vessel density, perfused vessel density, and microcirculatory flow index were assessed before and after the WB-NMES. Total blood vessel density was a measure of the total length of vessel divided by the total surface of the analyzed area. Perfused vessel density was the vessel density times the proportion of perfused vessels. The microcirculatory flow index was determined by dividing the average value after the microcirculation image into four quadrants and assigning a number according to the predominant flow type $(0=$ no flow, $1=$ intermittent, $2=$ sluggish, $3=$ continuous) [18].

\section{Derivatives of reactive oxygen metabolites and biological antioxidant potential}

Plasma levels of derivatives of reactive oxygen metabolites (d-ROMs) and biological antioxidant potential (BAP) were assessed using a d-ROMs test kit (Diacron, Grosseto, Italy) and BAP test (Diacron), respectively [19, 20]. The d-ROMs test depends on Fenton-like reactions, leading to the formation of lipid peroxy- and alkoxy-radicals that in turn react with a chromogenic substrate. The BAP test quantifies the body's reducing power to iron, which acts as a sensitive antioxidant.

\section{Blood lactate levels}

The blood lactate concentration was measured with the lactate oxidase method using an automated analyzer (Lactate Pro 2, Arkray, Kyoto, Japan) [21]. For this, $5 \mathrm{ml}$ of blood was obtained from the forearm vein before and after the WB-NMES.

\section{Echocardiography}

Echocardiographic examination was performed with a Philips HD 15 Pure Wave machine (Philips Medical System, USA) equipped with 2.5-3.5 MHz transducers. The left ventricular wall motion, left ventricular ejection fraction, left ventricular diastolic function (E/A, E/E'), 2D color Doppler imaging to detect mitral regurgitation and/or tricuspid regurgitation, and inferior vena cava diameter were assessed by echocardiography before and after WB-NMES. Echocardiographic monitoring was continued during WB-NMES.

\section{Study protocol}

Participants fasted for $5 \mathrm{~h}$ and abstained from drinking beverages containing alcohol or caffeine for $\geq 12 \mathrm{~h}$ before the study. All experiments were performed in a quiet, temperature-controlled room $\left(23-24{ }^{\circ} \mathrm{C}\right)$ on the same day. The volunteers were instructed to be in supine position. All the tests were supervised by a medical doctor. Echocardiography and 12-lead electrocardiogram (ECG) were performed on all participants before the WB-NMES (Fig. 1). Blood pressure and pulse rate were noninvasively measured every two min (HEM-6130-E, Omron Healthcare Co, Ltd, Kyoto, Japan). ECG monitoring (FCP-8800, Fukuda Denshi Co, Ltd, Tokyo, Japan) was continued during the study. Immediately after blood sampling and assessment of echocardiography, WB-NMES was applied for $20 \mathrm{~min}$ to the anterior and posterior upper arm, chest, back, abdominal, abdominal oblique, gluteus, and anterior and posterior thigh muscles using the custom-made stimulator based on a plot type battery-powered WB-NMES device (SIXPAD, MTG Ltd., Nagoya, Japan) (Fig. 2). Electrical stimulation
Fig. 1 Experimental protocol schedule. 12-lead electrocardiogram (ECG), Blood pressure, pulse rate, echocardiography, blood sampling and CytoCam test were performed on all participants before the whole body neuromuscular electrical stimulation (WB-NMES). ECG monitoring was continued during the study. Echocardiography confirmed wall motion and new appearance of valve during WBNMES. A 12-lead ECG, echocardiography, blood sampling and CytoCam test were repeated 10 min after termination of the WB-NMES

\begin{tabular}{lccc}
\hline $\begin{array}{l}\text { Type of } \\
\text { measurement }\end{array}$ & Baseline & $\begin{array}{c}\text { WB-NMES for 20 } \\
\text { min }\end{array}$ & $\begin{array}{c}\text { 10 min after WB-NMES } \\
\text { termination }\end{array}$ \\
\hline ECG monitoring & $\square$ & $\square$ & $\square$ \\
12-lead ECG & $\square$ & & $\square$ \\
Blood pressure & $\square$ & $\square$ & $\square$ \\
Pulse rate & $\square$ & $\square$ & $\square$ \\
$\begin{array}{l}\text { Echocardiography } \\
\text { Blood sampling }\end{array}$ & $\square$ & $\square$ & $\square$ \\
$\begin{array}{l}\text { CytoCam test to } \\
\text { evaluate sublingual } \\
\text { microcirculation }\end{array}$ & $\square$ & & $\square$ \\
\hline
\end{tabular}


(a)

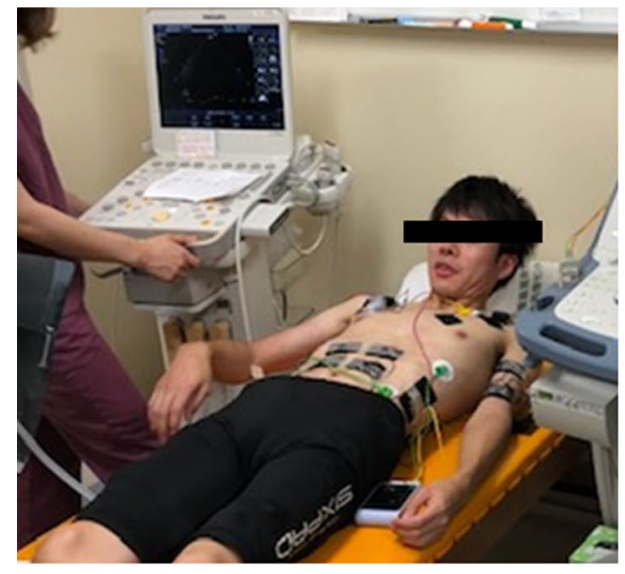

(d)

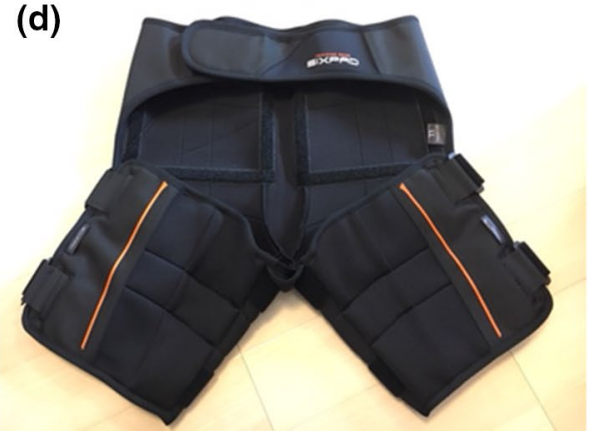

(b)

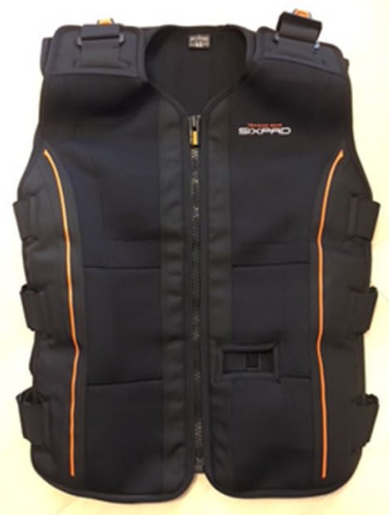

(c)

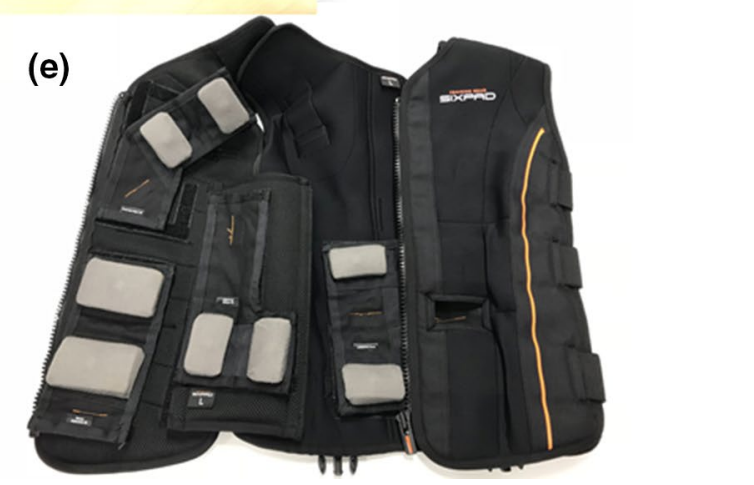

arm. d Shorts for WB-NMES. e The position of the electrodes is shown inside the vest. In chest, back, abdominal and abdominal oblique

\section{Results}

No arrhythmia was detected during the WB-NMES or after the $10 \mathrm{~min}$ recovery period. Blood pressure and heart rate remained unchanged during the WB-NMES and recovery period. Echocardiography showed that the left ventricular ejection fraction and diastolic function were maintained in all participants, but one showed transient mild mitral regurgitation during WB-NMES which lasted a few minutes after termination of WB-NMES (Table 2). Five of the 19 patients received electrical stimulation three times a week for 1 month, with no adverse effects observed.

Significant decreases in blood glucose and increases in lactate levels were observed (Fig. 3). Although there were no significant differences in d-ROMs, BAP, or blood fluidity (Table 3, Fig. 4), three out of 19 participants showed prolonged whole blood passage time just after WB-NMES. The whole blood passage time of these three participants recovered to baseline measures $20 \mathrm{~min}$ after WB-NMES (Fig. 5). The sublingual microcirculation parameters were not statistically different before and after WB-NMES (Table 3, Fig. 5). 
Table 2 Effects of wholebody neuromuscular electrical stimulation on hemodynamics, arrythmia, and echocardiographic parameters
Fig. 3 Effects of whole-body neuromuscular electrical stimulation (WB-NMES) on blood level of lactic acid (a) and glucose (b) shows that WBNMES significantly increased blood level of lactic acid (a) and significantly decreased blood glucose levels $(\mathbf{b})$

\begin{tabular}{|c|c|c|c|c|}
\hline & $\begin{array}{l}\text { Baseline } \\
(n=19)\end{array}$ & $\begin{array}{l}\text { During WB-NMES } \\
(n=19)\end{array}$ & $\begin{array}{l}10 \text { min After } \\
\text { WB-NMES } \\
(n=19)\end{array}$ & $P$-value \\
\hline Arrhythmia on ECG monitor (\%) & 0 & 0 & 0 & - \\
\hline Systolic blood pressure (mmHg) & $115.1 \pm 10.8$ & - & $119.3 \pm 10.4$ & $0.06^{\mathrm{a}}$ \\
\hline Diastolic blood pressure $(\mathrm{mmHg})$ & $72.6 \pm 9.6$ & - & $71.8 \pm 9.6$ & $0.6^{\mathrm{b}}$ \\
\hline Heart rate (beats per min) & $71.4 \pm 9.7$ & - & $73.2 \pm 7.4$ & $0.3^{\mathrm{a}}$ \\
\hline $\operatorname{LVEF}(\%)$ & $60.3 \pm 2.8$ & $60.2 \pm 2.9$ & $61.0 \pm 2.7$ & $0.4^{\mathrm{c}}$ \\
\hline $\mathrm{E} / \mathrm{A}$ ratio & $1.7 \pm 0.2$ & $1.6 \pm 0.2$ & $1.6 \pm 0.2$ & $0.3^{\mathrm{d}}$ \\
\hline Mean E/e' & $6.1 \pm 1.6$ & $6.3 \pm 1.4$ & $6.1 \pm 1.2$ & $0.9^{\mathrm{d}}$ \\
\hline $\operatorname{IVC}(\mathrm{cm})$ & $0.8 \pm 0.3$ & $0.6 \pm 0.3$ & $0.8 \pm 0.2$ & $0.07^{\mathrm{d}}$ \\
\hline Occurrence of TR (\%) & 0 & 0 & 0 & - \\
\hline Occurrence of MR (\%) & 0 & $1(5.3)$ & 0 & - \\
\hline
\end{tabular}

Data are expressed as the mean $\pm \mathrm{SD}$, or number $(\%)$

${ }^{\text {a S}}$ Student's $t$-test

${ }^{\mathrm{b}}$ Wilcoxon signed rank test

${ }^{\mathrm{c}}$ One-way repeated measures analysis of variance

${ }^{\mathrm{d}}$ Friedman test

$A$ atrial systolic wave, $E$ early diastolic wave, $e$ ' early diastolic wall motion velocity, $E C G$ electrocardiogram, $I V C$ inferior vena cava, $L V E F$ left ventricular ejection fraction, $M R$ mitral regurgitation, $T R$ tricuspid regurgitation
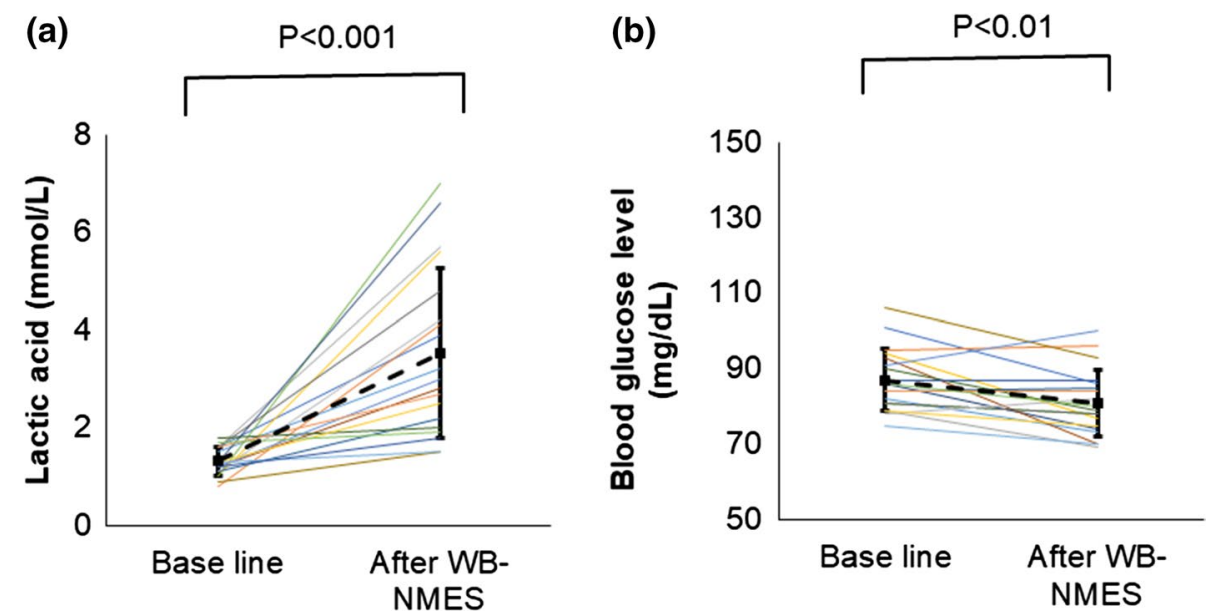

\section{Discussion}

This study aimed to investigate the safety and effectiveness of WB-NMES on hemodynamics, arrhythmia, and sublingual microcirculation. Our results from healthy participants showed that no arrhythmia was detected, while the WB-NMES effectively increased blood lactate level, decreased blood glucose level, and maintained blood pressure, blood fluidity, and sublingual microcirculation.

WB-NMES has been introduced as an alternative to physical training or in combination with low-grade exercise. However, associated muscular damage, as evident by elevation of serum creatine-kinase activity, has been reported [23, 24]. Until more evidence regarding the physiological contraindications is gathered $[8,24]$, the electrical strength should be prescribed, the health of the users should be considered, and instructors should be trained to avoid WB-NMES-related side effects [25].

Although the efficacy and safety of WB-NMES have been examined, specific guidelines regarding cardiopulmonary function are lacking. A study by Jee et al. [26] reported that there were no abnormal changes in heart rate, systolic and diastolic blood pressure, and oxygen uptake during or after WB-NMES. The major findings of the present study suggest that the application of the maximum tolerable WB-NMES intensity did not cause arrhythmias and did not negatively affect heart rate, systolic and diastolic blood pressure, or 
Table 3 Effects of wholebody neuromuscular electrical stimulation on blood parameters and whole blood passage time
Fig. 4 Effects of whole-body neuromuscular electrical stimulation (WB-NMES) on blood level of diacron-reactive oxygen metabolites (a) and biological antioxidant potential (b) as antioxidant power. The blood level of diacron-reactive oxygen metabolites (a) and biological antioxidant potential (b) remains unchanged by WBNMES

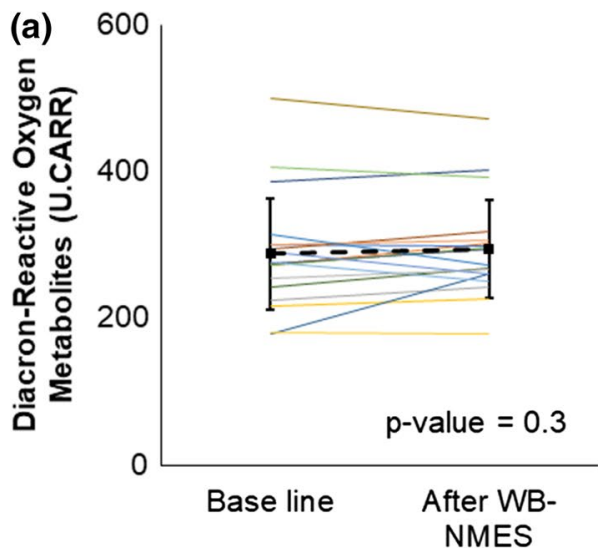

(a)

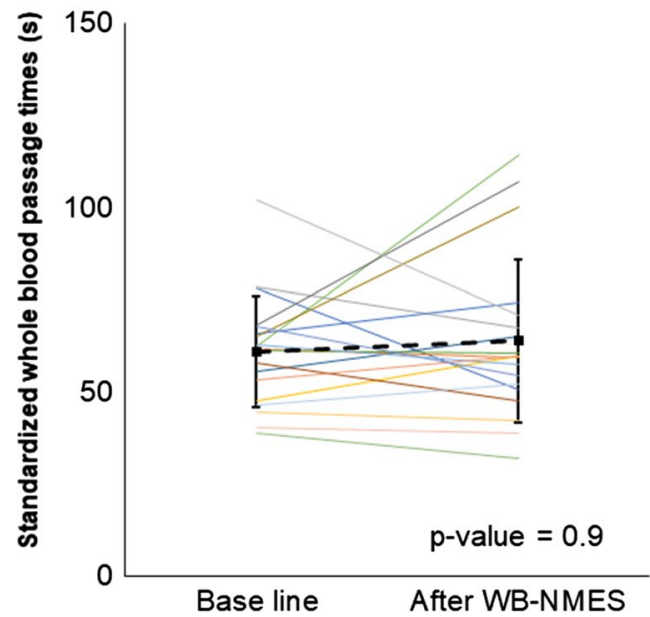

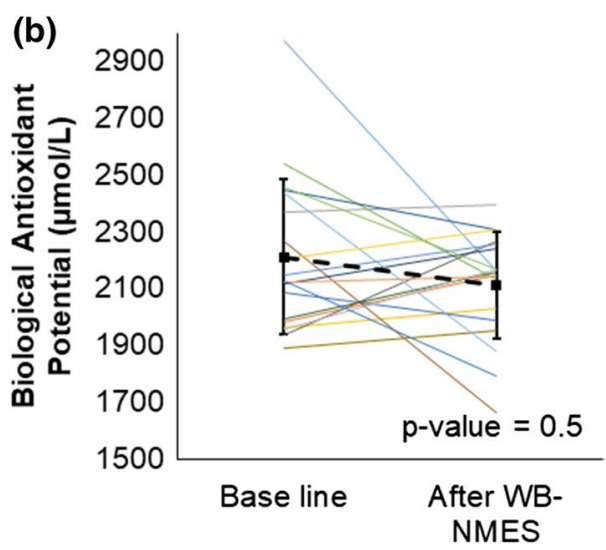

(b)

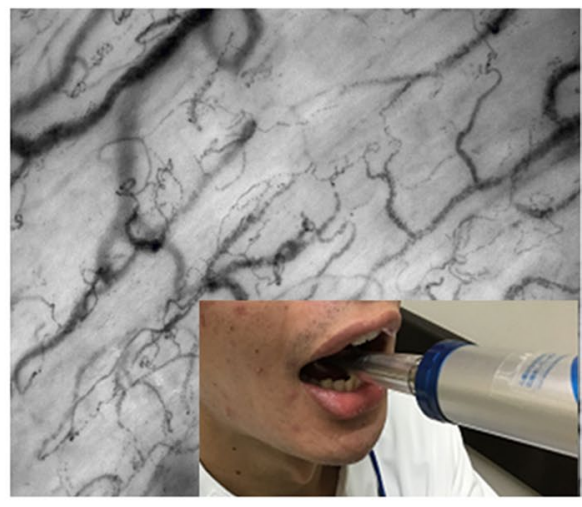

Fig. 5 a Effects of wholebody neuromuscular electrical stimulation on whole blood passage time as hemorheology and sublingual microcirculation parameters. Whole blood passage time remains unchanged by whole-body neuromuscular electrical stimulation (WBNMES). b A sublingual microcirculation image was captured using a handheld dark-field CytoCam video microscope before and after WB-NMES
Lactic acid (mmol/L)

Blood glucose level (mg/dL)

Noradrenaline $(\mathrm{pg} / \mathrm{mL})$

Hematocrit (\%)

Diacron-reactive oxygen metabolites (U.CARR)

Biological antioxidant potential $(\mu \mathrm{mol} / \mathrm{L})$

Whole blood passage time (s)

Sublingual total blood vessel density $\left(\mathrm{mm} / \mathrm{mm}^{2}\right)$

Sublingual perfused vessel density $\left(\mathrm{mm} / \mathrm{mm}^{2}\right)$

Sublingual microcirculatory flow index

Data are expressed as the mean $\pm \mathrm{SD}$ (standard deviation), or number (\%)

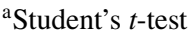

${ }^{\mathrm{b}}$ Wilcoxon signed rank test

U.CARR Carratelli unit, $1 \mathrm{U} . \mathrm{CARR}=0.08 \mathrm{mg} \mathrm{H}_{2} \mathrm{O}_{2} / \mathrm{dL}$

\begin{tabular}{lll}
$\begin{array}{l}\text { Baseline } \\
(n=19)\end{array}$ & $\begin{array}{l}10 \text { min after WB-NMES } \\
(n=19)\end{array}$ & $P$-value \\
\hline $1.3 \pm 0.3$ & $3.5 \pm 1.8$ & $0.00004^{\mathrm{a}}$ \\
$87.0 \pm 8.1$ & $80.8 \pm 9.0$ & $0.004^{\mathrm{a}}$ \\
$0.2 \pm 0.1$ & $0.2 \pm 0.1$ & $0.4^{\mathrm{a}}$ \\
$43.5 \pm 3.2$ & $43.1 \pm 3.7$ & $0.4^{\mathrm{b}}$ \\
$287.2 \pm 76.7$ & $294.7 \pm 66.6$ & $0.3^{\mathrm{b}}$ \\
$2212.2 \pm 271.2$ & $2114.4 \pm 188.6$ & $0.5^{\mathrm{b}}$ \\
$60.6 \pm 15.4$ & $64.5 \pm 22.6$ & $0.9^{\mathrm{b}}$ \\
$10.4 \pm 2.2$ & $10.3 \pm 2.9$ & $0.8^{\mathrm{a}}$ \\
$6.9 \pm 2.0$ & $6.1 \pm 3.1$ & $0.3^{\mathrm{b}}$ \\
$2.6 \pm 0.3$ & $2.5 \pm 0.8$ & $0.2^{\mathrm{b}}$
\end{tabular}


function with echocardiography. We found transient mild mitral regurgitation in one male subject during WB-NMES. The volume of transient functional mitral regurgitation was too small to measure precisely. Vena contracta at the suction was $1.0 \mathrm{~mm}$ with colour area trace method. And his cardiac function was within the normal range. The possible mechanism may be a temporary increase in venous perfusion due to WB-NMES, because the mitral regurgitation was observed during lower extremity NMES without pulmonary hypertension. Lower extremity NMES increases venous return in normal participants and patients with heart disease. The activation of the muscular-venous pump by NMES-induced muscle contraction increases venous return [27]. For this reason, patients with NYHA class IV heart failure (left ventricular ejection fraction $<25 \%$ ), severe cardiac arrhythmias, and electronic devices such as pacemakers and implanted defibrillators, are listed as contraindications in WB- NMES trials [24-26]. Exercise-induced pulmonary hypertension in patients with secondary mitral regurgitation is a powerful predictor of poor outcomes, with increased risk for cardiac-related death [28]. Therefore, the safety and effects of WB-NMES in patients with heart failure is a target for future research.

Blood glucose was significantly decreased by WB-NMES in this study. This is similar to findings from a previous study [6] that found insulin-independent glucose uptake during muscle contraction when NMES was performed on the lower legs. NMES to the lower legs has been reported to significantly enhance whole-body glucose uptake compared to voluntary ergometry exercise at the identical oxygen uptake in the presence of significantly higher blood lactate and respiratory quotients [6]. The reduction in glucose after NEMS is most likely due to insulin-independent glucose transporter type 4 upload [29]. Although electric stimulation was applied to several regions of the body in the present study, it was not clear whether it had a stronger hypoglycemic effect than general NMES devices. However, it is known that NMES can enhance anaerobic energy metabolism due to the recruitment of high-threshold motor units or muscle fibers associated with glucose metabolism [6,30].

Lactate accumulates during anaerobic exercise and can be utilized as an energy source by skeletal muscles during voluntary exercise [31]. Lactic acid increased from $1.3 \pm 0.3 \mathrm{mmol} / \mathrm{L}$ at rest to $3.5 \pm 1.8 \mathrm{mmol} / \mathrm{L}$ after $\mathrm{WB}-$ NMES. In the present study, it was not clear if the maximum effect was reached by applying electrical stimulation over a wider range compared to the lower leg alone. In our pilot study, three participants showed $2.96 \pm 0.3$ metabolic equivalents (METs) on the cardiopulmonary exercise test during WB-NMES alone. In a previous study in which NMES was applied to the lower leg, the exercise intensity showed 3.0 \pm 0.47 METs [32], which is similar to our results. Therefore, these observations suggest that lactate production from skeletal muscle is limited during walking equivalent to three METs, whereas is much higher with WB-NMES at three METs. In addition, aerobic exercise and resistance training increased blood pressure and heart rate, while WB-NMES alone did not increase either. Therefore, we posit that WBNMES can provide the systemic skeletal muscle a relatively high-intensity exercise load that produces lactate without affecting cardiac function. In addition, Watanabe et al. [10] have reported that combining WB-NMES with low-intensity voluntary exercise lead greater energy expenditure with the metabolic equivalent of $5.31 \pm 0.89$ Mets and serum lactic acid level of $7.5 \mathrm{mmol} / \mathrm{L}$. They concluded that more lactic acid was produced by combining voluntary exercise and WB-NMES than WB-NMES alone [10].

Blood fluidity was not significantly different before and after WB-NMES in the present study. Several studies have reported that acute exercise at the anaerobic threshold reduces blood fluidity $[14,33]$. The decrease is explained by the increased blood viscosity due to the increase of hematocrit induced by dehydration and activation of white blood cells [14], platelets, and cytokine [13]. However, vigorous exercise leads to increased oxidative stress [34], which may be associated with a decrease in insulin secretory ability and aggravates diabetes [35]. An increase in oxidative stress contributes to the development of various diseases such as cancer, atherosclerosis, and aging [36]. The present results showed that d-ROMs and BAP remained unchanged by WB-NMES.

There were limitations to the study to consider. First, the sample size was limited, because the present study was a single-center observational study analysis without control, and thus, causation could not be established. However, the number of 19 participants may be appropriate as a modified phase 1 study. Second, the study participants were limited to healthy young men and women, so the results cannot be applied to other populations. Third, as we gave only one 20-min session of electrical stimulation, we cannot comment on the safety and efficacy of its long-term use.

\section{Conclusion}

WB-NMES applied to healthy young individuals with the tolerable maximum intensity for 20 min significantly decreased blood glucose and increased blood lactate level; however, it did not change hemodynamic parameters, and it did not induce arrythmia or changes in blood fluidity and sublingual microcirculation. The effects of combining resistant training and/or stretching with WB-NMES should be clarified in future studies. In addition, safety and efficacy of WB-NMES in patients with chronic heart disease or chronic respiratory failure should be examined. 
Acknowledgements The authors thank Prof. Toshio Moritani and Dr. Toshiaki Miyamoto for their critical advice on this manuscript. We also thank Keiko Yoshizawa for administrative assistance and Nozomi Saito for her technical assistant. We would like to thank Editage (www.edita ge.com) for English language editing.

Funding This work was supported by a Grant from MTG Co. Ltd (to TY), by Dokkyo Medical University, Young Investigator Award (No.2018-23) (to MH) and by the Vehicle Racing Commemorative Foundation (to TY).

Data availability The deidentified participant data will not be shared.

\section{Compliance with ethical standards}

Conflict of interest This work was supported by a Grant from MTG Co. Ltd. After fixation of the database, the data and results of analyses were reported to MTG Ltd. MTG Co. Ltd. was not involved in the implementation, analysis, or reporting of this study. Dr. Yasu reports receiving grant support and lecture fees from AstraZeneca K.K., Ono Pharmaceutical Co., Ltd., and Kowa Co. Ltd, grant support from MTG Co. Ltd., lecture fees from Actelion Pharmaceuticals Ltd, Sanofi S.A., Daiichi Sankyo Co, Ltd, and Takeda Pharmaceutical Co. Ltd. There are no other conflicts of interest of each author.

Ethics approval This study was approved by our institutional ethics committee (Approval Number: Nikko 29012). All procedures performed in this study complied with the national ethical guidelines for medical and health research involving human participants and with the 1964 Helsinki Declaration and its later amendments or comparable ethical standards.

Informed consent to participate Written informed consent was obtained from the participants.

Informed consent to publish The authors affirm that human research participants provided informed consent for publication.

Open Access This article is licensed under a Creative Commons Attribution 4.0 International License, which permits use, sharing, adaptation, distribution and reproduction in any medium or format, as long as you give appropriate credit to the original author(s) and the source, provide a link to the Creative Commons licence, and indicate if changes were made. The images or other third party material in this article are included in the article's Creative Commons of microvessels to assess the whole blood rheology andlicence, unless indicated otherwise in a credit line to the material. If material is not included in the article's Creative Commons licence and your intended use is not permitted by statutory regulation or exceeds the permitted use, you will need to obtain permission directly from the copyright holder. To view a copy of this licence, visit http://creativecommons.org/licenses/by/4.0/.

\section{References}

1. Tashiro H, Tanaka A, Ishii H, Motomura N, Arai K, Adachi T, Okajima T, Iwakawa N, Kojima H, Mitsuda T, Hirayama K, Hitora Y, Hayashi M, Furusawa K, Yoshida R, Imai H, Ogawa Y, Kawaguchi K, Murohara T (2020) Reduced exercise capacity and clinical outcomes following acute myocardial infarction. Heart Vessels 35:1044-1050
2. Hambrecht R, Fiehn E, Yu J, Niebauer J, Weigl C, Hilbrich L, Adams V, Riede U, Schuler G (1997) Effects of endurance training on mitochondrial ultrastructure and fiber type distribution in skeletal muscle of patients with stable chronic heart failure. J Am Coll Cardiol 29:1067-1073

3. Schulze PC, Gielen S, Schuler G, Hambrecht R (2002) Chronic heart failure and skeletal muscle catabolism: effects of exercise training. Int J Cardiol 85:141-149

4. Kitamura M, Izawa KP, Ishihara K, Yaekura M, Nagashima H, Yoshizawa T, Okamoto N (2020) Predictors of activities of daily living at discharge in elderly patients with heart failure with preserved ejection fraction. Heart Vessels. https://doi.org/10.1007/ s00380-020-01718-6

5. Dobsák P, Nováková M, Siegelová J, Fiser B, Vítovec J, Nagasaka M, Kohzuki M, Yambe T, Nitta S, Eicher JC, Wolf JE, Imachi K (2006) Low-frequency electrical stimulation increases muscle strength and improves blood supply in patients with chronic heart failure. Circ J 70:75-82

6. Hamada T, Hayashi T, Kimura T, Nakao K, Moritani T (2004) Electrical stimulation of human lower extremities enhances energy consumption, carbohydrate oxidation, and whole body glucose uptake. J Appl Physiol 96:911-916

7. Hamada T, Sasaki H, Hayashi T, Moritani T, Nakao K (2003) Enhancement of whole body glucose uptake during and after human skeletal muscle low-frequency electrical stimulation. J Appl Physiol 94:2107-2112

8. Hasegawa S, Kobayashi M, Arai R, Tamaki A, Nakamura T, Moritani T (2011) Effect of early implementation of electrical muscle stimulation to prevent muscle atrophy and weakness in patients after anterior cruciate ligament reconstruction. J Electromyogr Kinesiol 21:622-630

9. Moritani T, Muro M, Kijima A (1985) Electromechanical changes during electrically induced and maximal voluntary contractions: electrophysiologic responses of different muscle fiber types during stimulated contractions. Exp Neurol 88:471-483

10. Watanabe K, Yoshida T, Ishikawa T, Kawade S, Moritani T (2019) Effect of the combination of whole-body neuromuscular electrical stimulation and voluntary exercise on metabolic responses in human. Front Physiol 10:291

11. Lowe G, Rumley A, Norrie J, Ford I, Shepherd J, Cobbe S, Macfarlane P, Packard C (2000) Blood rheology, cardiovascular risk factors, and cardiovascular disease: the West of Scotland Coronary Prevention Study. Thromb Haemost 84:553-558

12. El-Sayed MS, Ali N, El-Sayed Ali Z (2005) Haemorheology in exercise and training. Sports Med 35:649-670

13. El-Sayed MS (1998) Effects of exercise and training on blood rheology. Sports Med 26:281-292

14. Ikeda N, Yasu T, Tsuboi K, Sugawara Y, Kubo N, Umemoto T, Arao K, Kawakami M, Momomura S (2010) Effects of submaximal exercise on blood rheology and sympathetic nerve activity. Circ J 74:730-734

15. Fukuda S, Yasu T, Predescu DN, Schmid-Schönbein GW (2000) Mechanisms for regulation of fluid shear stress response in circulating leukocytes. Circ Res 86:E13-E18

16. Yasu T, Kobayashi M, Mutoh A, Yamakawa K, Momomura S, Ueda S (2013) Dihydropyridine calcium channel blockers inhibit non-esterified-fatty-acid-induced endothelial and rheological dysfunction. Clin Sci (Lond) 125:247-255

17. Yasu T, Mutoh A, Wada H, Kobayashi M, Kikuchi Y, Momomura S, Ueda S (2018) Renin-angiotensin system inhibitors can prevent intravenous lipid infusion-induced myocardial microvascular dysfunction and leukocyte activation. Circ J 82:494-501

18. Donati A, Damiani E, Domizi R, Romano R, Adrario E, Pelaia P, Ince C, Singer M (2013) Alteration of the sublingual microvascular glycocalyx in critically ill patients. Microvasc Res 90:86-89 
19. Cornelli U, Terranova R, Luca S, Cornelli M, Alberti A (2001) Bioavailability and antioxidant activity of some food supplements in men and women using the D-Roms test as a marker of oxidative stress. J Nutr 131:3208-3211

20. Vassalle C, Boni C, Di Cecco P, Landi P (2006) Elevated hydroperoxide levels as a prognostic predictor of mortality in a cohort of patients with cardiovascular disease. Int J Cardiol 110:415-416

21. Watanabe K, Taniguchi Y, Moritani T (2014) Metabolic and cardiovascular responses during voluntary pedaling exercise with electrical muscle stimulation. Eur J Appl Physiol 114:1801-1807

22. Filipovic A, Kleinöder H, Dörmann U, Mester J (2011) Electromyostimulation-a systematic review of the influence of training regimens and stimulation parameters on effectiveness in electromyostimulation training of selected strength parameters. J Strength Cond Res 25:3218-3238

23. Stöllberger C, Finsterer J (2019) Side effects of whole-body electro-myo-stimulation. Wien Med Wochenschr 169:173-180

24. Stöllberger C, Finsterer J (2019) Side effects of and contraindications for whole-body electro-myo-stimulation: a viewpoint. BMJ Open Sport Exerc Med 5:e000619

25. Kemmler W, Froehlich M, von Stengel S, Kleinöder H (2016) Whole-Body electromyostimulation - the need for common sense! Rationale and guideline for a safe and effective training. Dtsch Z Sportmed 67:218-221

26. Jee YS (2018) The efficacy and safety of whole-body electromyostimulation in applying to human body: based from graded exercise test. J Exerc Rehabil 14:49-57

27. Wm IO, Lepar GS, Morrissey MC, Cywinski JK (2003) Effect of neuromuscular electrical stimulation on foot/ankle volume during standing. Med Sci Sports Exerc 35:630-634

28. Lancellotti P, Magne J, Dulgheru R, Ancion A, Martinez C, Piérard LA (2015) Clinical significance of exercise pulmonary hypertension in secondary mitral regurgitation. Am J Cardiol 115:1454-1461

29. Miyamoto T, Fukuda K, Kimura T, Matsubara Y, Tsuda K, Moritani T (2012) Effect of percutaneous electrical muscle stimulation on postprandial hyperglycemia in type 2 diabetes. Diabetes Res Clin Pract 96:306-312
30. Hamada T, Kimura T, Moritani T (2004) Selective fatigue of fast motor units after electrically elicited muscle contractions. J Electromyogr Kinesiol 14:531-538

31. Brooks GA (1986) The lactate shuttle during exercise and recovery. Med Sci Sports Exerc 18:360-368

32. Minogue CM, Caulfield BM, Lowery MM (2014) Whole body oxygen uptake and evoked torque during subtetanic isometric electrical stimulation of the quadriceps muscles in a single 30-minute session. Arch Phys Med Rehabil 95:1750-1758

33. Haskell WL, Lee IM, Pate RR, Powell KE, Blair SN, Franklin BA, Macera CA, Heath GW, Thompson PD, Bauman A (2007) Physical activity and public health: updated recommendation for adults from the American College of Sports Medicine and the American Heart Association. Circulation 116:1081-1093

34. Pingitore A, Lima GP, Mastorci F, Quinones A, Iervasi G, Vassalle C (2015) Exercise and oxidative stress: potential effects of antioxidant dietary strategies in sports. Nutrition 31:916-922

35. Kumashiro N, Tamura Y, Uchida T, Ogihara T, Fujitani Y, Hirose T, Mochizuki H, Kawamori R, Watada H (2008) Impact of oxidative stress and peroxisome proliferator-activated receptor gamma coactivator-1alpha in hepatic insulin resistance. Diabetes 57:2083-2091

36. Akatsuka S, Aung TT, Dutta KK, Jiang L, Lee WH, Liu YT, Onuki J, Shirase T, Yamasaki K, Ochi H, Naito Y, Yoshikawa T, Kasai H, Tominaga Y, Sakumi K, Nakabeppu Y, Kawai Y, Uchida K, Yamasaki A, Tsuruyama T, Yamada Y, Toyokuni S (2006) Contrasting genome-wide distribution of 8-hydroxyguanine and acrolein-modified adenine during oxidative stress-induced renal carcinogenesis. Am J Pathol 169:1328-1342

Publisher's Note Springer Nature remains neutral with regard to jurisdictional claims in published maps and institutional affiliations. 